\title{
The Calculation of Fourier Coefficients by the Möbius Inversion of the Poisson Summation Formula Part II. Piecewise Continuous Functions and Functions with Poles near the Interval $[0,1]^{*}$
}

\author{
By J. N. Lyness
}

\begin{abstract}
In Part I, the MIPS method for calculating Fourier coefficients was introduced, and applied to functions $f \in C^{(p)}[0,1]$. In this part two extensions of the theory are described.

One modification extends the theory to piecewise continuous functions, $f \in P C^{(p)}[0,1]$. Using these results the method may be used to calculate approximations to trigonometrical integrals (in which the length of the interval need not coincide with a period of the trigonometrical weighting function).

The other modification treats functions which are analytic, but whose low-order derivatives vary rapidly due to poles in the complex plane near the interval of integration. Essentially these poles are 'subtracted out' but this is done implicitly by the inclusion of additional terms in the standard series.

The practical application of these modified methods requires that the nature and location of the discontinuities - or poles-be known at least approximately.
\end{abstract}

1. Introduction. In Part I (Lyness [2]) several representations for Fourier coefficients

$$
C^{(m)} f=\int_{0}^{1} f(x) \cos 2 \pi m x d x, \quad S^{(m)} f=\int_{0}^{1} f(x) \sin 2 \pi m x d x
$$

are derived. These express a Fourier coefficient as the sum of the first several terms of its standard asymptotic expansion together with a remainder term which involves trapezoidal rule sums such as

$$
R^{|m, 1|} f=\frac{1}{m} \sum_{j=0}^{m}{ }^{\prime \prime} f\left(\frac{j}{m}\right) .
$$

One of these representations (Part I, Eq. (7.2)) is

$$
2 C^{(m)} f=\sum_{a=1}^{n} \frac{\tilde{K}_{2 a}}{m^{2 a}}+\sum_{s=1}^{\infty} \mu_{s} \tilde{E}_{2 n+2}^{(m s, 1)} f,
$$

where

$$
\tilde{E}_{2 n+2}^{[m, 1]} f=R^{[m, 1]} f-\int_{0}^{1} f(x) d x-\sum_{q=1}^{n} \frac{\tilde{K}_{2 q} \zeta(2 q)}{m^{2 q}},
$$

Received May 15, 1970, revised August 17, 1970.

AMS 1969 subject classifications. Primary 4210, 6555; Secondary 4208.

Key words and phrases. Fourier coefficients, Euler-Maclaurin summation formula, Fourier coefficient asymptotic expansion, numerical quadrature, subtracting out singularities.

* Work performed under the auspices of the United States Atomic Energy Commission. 
$\tilde{K}_{2 q}$ is arbitrary, and $\mu_{s}$ are Möbius numbers. This formula is valid for all functions $f(x)$ which are continuous in the closed interval $[0,1]$, but is useful if $f(x) \in C^{(2 n+2)}[0,1]$. In this case the ultimate convergence rate of the sum over index $s$ is optimum if $\tilde{K}_{2 a}$ coincides with $K_{2 q}$ given by

$$
K_{2 q}=2(-1)^{a-1}\left(f^{(2 q-1)}(1)-f^{(2 q-1)}(0)\right) /(2 \pi)^{2 q} .
$$

Two other representations for $C^{(m)} f$ of a type similar to (1.3) are given in Part I together with one for $S^{(m)} f$. The practical use of (1.3) as a basis for computation involves calculating members of the sequence

$$
\tilde{E}_{2 n+2}^{[s, 1]} f, \quad s=1,2,3, \cdots,
$$

for a fixed $n$, and terminating this calculation after some evaluation $s=\bar{s}$. In practice the choice of $\bar{s}$ should be made in such a way that the user is confident that

$$
\sum_{s=s+1}^{\infty}\left|\tilde{E}_{2 n+2}^{[s, 1]} f\right|<2 \epsilon,
$$

where $\epsilon$ is the required tolerance. The approximations $\tilde{C}^{(m)} f$ are then obtained by setting

$$
\widetilde{E}_{2 n+2}^{[s, 1]} f=0, \quad s>\bar{s},
$$

in (1.3) wherever such terms occur. If $\bar{s}$ satisfies (1.7) then the resulting approximation $\tilde{C}^{(m)} f$ satisfies

$$
\left|\tilde{C}^{(m)} f-C^{(m)} f\right|<\epsilon .
$$

The theory of which all these equations form part is fully described in Part I.

Methods based on these formulas are termed MIPS methods since these formulas may be derived by means of "Möbius Inversion of the Poisson Summation Formula." These methods generally suffer from the drawback that approximations to the derivatives of $f(x)$ at $x=0$ and $x=1$ are required, but they have the advantage of providing naturally a set of results with uniform absolute accuracy.

In common with many other computational techniques, there exist classes of problems for which the standard MIPS method does not work efficiently. In some cases the method may be specially adapted to deal with such problems. In this paper we derive two such modifications. These are designed for the following problems:

(i) $f(x)$ is piecewise continuous, i.e., $f \in P C^{(p)}[0,1]$. Thus, with $0=x_{0}<x_{1}<$ $x_{2} \cdots<x_{n}=1$,

$$
\begin{aligned}
f(x) & =\phi_{i}(x) \text { on } x_{i-1}<x<x_{i}, & & i=1,2, \cdots, n, \\
f\left(x_{i}\right) & =\frac{1}{2}\left(\phi_{i-1}\left(x_{i}\right)+\phi_{i}\left(x_{i}\right)\right), & & i=1,2, \cdots, n-1, \\
f(0) & =f(1)=\frac{1}{2}\left(\phi_{n}\left(x_{n}\right)+\phi_{1}\left(x_{0}\right)\right), & &
\end{aligned}
$$

and each function $\phi_{i}(x)$ is $C^{p}\left[x_{i-1}, x_{i}\right]$.

(ii) $f(x)$ has high peaks due to nearby poles in the complex plane. An example of such a function is

$$
f(x)=\theta(x) /\left(x^{2}-2 \lambda x+\lambda^{2}+\mu^{2}\right)
$$

where $0<\lambda<1$ and $|\mu|$ is smaller than both $\lambda$ and $1-\lambda$. 
It turns out that in both cases there is a relatively straightforward way to adjust the formulas involved to take direct account of discontinuities and poles. This may be done within the framework of the existing method. Additional terms of a specified nature have to be included in formulas (1.3) and (1.4) or existing terms have to be modified.

This paper is organized in the following way. Piecewise continuous functions ((i) above) are treated in Sections 2 and 3 and, quite independently; functions with nearby poles ((ii) above) are treated in Sections 4, 5, and 6. Sections 2 and 5 are devoted to the mathematical derivation of the various formulas while Sections 3 and 6 discuss points that arise in their practical application.

2. Piecewise Continuous Functions: Theory. The formulas to be derived in this paper are all linear in the integrand function $f(x)$. Consequently in treating a function like (1.10) which takes a different form on different subintervals, it is notationally convenient to carry out a detailed calculation for a single typical subinterval. Subsequently (see Section 3(i)), formulas valid for the more general piecewise continuous function (1.10) may be obtained by combining formulas for each subinterval.

In this section, then, we deal specifically with deriving formulas for the calculation of a set of Fourier coefficients corresponding to a function $f(x)$ which is nonzero only in a subinterval $[a, b]$ of the interval $[0,1]$. In the open subinterval $(a, b), f(x)$ coincides with a given function $\phi(x)$ which satisfies

$$
\phi(x) \in C^{p}[a, b] .
$$

In addition we require $f(x)$ to coincide with $\bar{f}(x)$, the sum of its Fourier series in $(0,1)$. These restrictions lead to the following definitions:

Generally (unless both $a=0$ and $b=1$ )

$$
\begin{array}{ll}
f(x)=\phi(x) & \text { for } a<x<b, \\
f(x)=\frac{1}{2} \phi(x) & \text { for } x=a, \quad x=b, \\
f(x)=0 & \text { for other values of } x \text { in interval }[0,1] .
\end{array}
$$

In the special case that $a=0$ and $b=1$

$$
\begin{aligned}
& f(x)=\phi(x) \quad \text { for } 0<x<1, \\
& f(0)=f(1)=\frac{1}{2}(\phi(0)+\phi(1)) .
\end{aligned}
$$

The special case (2.2b) is, of course, the case considered in Part I, and the relevant results are given there. It is mentioned here only to draw attention to the slightly different structure which has to be taken into account if results given here are used with $a=0$ and $b=1$.

We derive a formula which is analogous to (1.3) above and which reduces to (1.3) in the case $a=0, b=1$. In order to do this we require a special form of the Euler-Maclaurin formula. This is obtained following the general method given in Sections 2 and 3 of Part I. The results of Part I, Section 2 are equally valid here since $f(x)$ as defined above is a function which coincides with its Fourier series, i.e., $\bar{f}(x)=f(x)$. As in Section 2 we define the off-set trapezoidal rule by

$$
R^{\lfloor m, \alpha]} f=\frac{1}{m} \sum_{j=1}^{m} f\left(\frac{j-1+t_{\alpha}}{m}\right), \quad t_{\alpha}=(1+\alpha) / 2, \quad|\alpha|<1 .
$$


The corresponding definition with $\alpha=1$ is (1.2) above. We also employ the notation

$$
\text { If }=\int_{0}^{1} f(x) d x .
$$

The Poisson summation formula (derived in Part I, Section 2) states that

$$
R^{[m, \alpha]} \bar{f}-I \bar{f}=2 \sum_{r=1}^{\infty} \cos 2 \pi r t_{\alpha} C^{(r m)} f+2 \sum_{r=1}^{\infty} \sin 2 \pi r t_{\alpha} S^{(r m)} f
$$

However the Fourier coefficient asymptotic expansions for $C^{(m)} f$ and $S^{(m)} f$ given in Part I, Section 3, Eqs. (3.3) and (3.4) are no longer valid except in the trivial cases $p=0$ and $p=-1$ respectively, since $f(x)$ is not continuous. Instead of these forms, we derive corresponding forms for the interval $[a, b]$. The derivation is iterative, using integration by parts at each stage. Thus

$$
\begin{aligned}
\int_{0}^{1} f(x) e^{i k x} d x & =\int_{a}^{b} \phi(x) e^{i k x} d x \\
& =\frac{i\left(-e^{i k b} \phi(b)+e^{i k a} \phi(a)\right)}{k}+\frac{i}{k} \int_{a}^{b} \phi^{\prime}(x) e^{i k x} d x .
\end{aligned}
$$

Iterating $p-1$ times, setting $k=2 \pi m$ and retaining only the real part leads to the formula

$$
\begin{aligned}
C^{(m)} f= & \sum_{a=1}^{p-1} \frac{-1}{(2 \pi m)^{a}} \\
& \cdot\left(\phi^{(a-1)}(b) \cos (2 \pi m b+q \pi / 2)-\phi^{(a-1)}(a) \cos (2 \pi m a+q \pi / 2)\right)+C_{p}^{(m)} f .
\end{aligned}
$$

Retaining the imaginary part instead gives a precisely analogous formula for $S^{(m)} f$ with sin replacing cos. The remainder terms are the real and imaginary parts of

$$
C_{p}^{(m)} f+i S_{p}^{(m)} f=\left(\frac{i}{2 \pi m}\right)^{p-1} \int_{a}^{b} \phi^{(p-1)}(x) e^{2 \pi i m x} d x .
$$

Since $\phi^{(p)}(x)$ is bounded, a single application of the second equality in (2.6) shows that these terms satisfy:

$$
C_{p}^{(m)} f \sim O\left(m^{-p}\right), \quad S_{p}^{(m)} f \sim O\left(m^{-p}\right) \quad \text { as } m \rightarrow \infty
$$

and are zero if $\phi(x)$ is a polynomial of degree $p-2$ or less. We now replace $C^{(r m)} f$ and $S^{(r m)} f$ in the Poisson summation formula (2.5) by the expression (2.7). The summation over index $r$ may be carried out in terms of Bernoulli functions, defined by

$$
\bar{B}_{q}(x)=-2 q ! \sum_{r=1}^{\infty} \frac{\cos (2 \pi r x-\pi q / 2)}{(2 \pi r)^{q}}, \quad q=0,1,2, \cdots .
$$

In this way we find

Euler-Maclaurin expansion for function (2.2).

$$
\begin{aligned}
R^{|m, \alpha|} f-I f= & \sum_{a=1}^{p-1} \frac{1}{m^{q}} \\
& \cdot\left\{\frac{\bar{B}_{q}\left(t_{\alpha}-m b\right)}{q !} \phi^{(q-1)}(b)-\frac{\bar{B}_{q}\left(t_{\alpha}-m a\right)}{q !} \phi^{(q-1)}(a)\right\}+E_{p}^{[m, \alpha]} f,
\end{aligned}
$$


where the remainder term is

$$
E_{p}^{[m, \alpha]} f=2 \sum_{r=1}^{\infty} \cos 2 \pi r t_{\alpha} C_{p}^{(r m)} f+2 \sum_{r=1}^{\infty} \sin 2 \pi r t_{\alpha} S_{p}^{(r m)} f
$$

In view of the properties of the remainder terms $C_{p}^{(m)} f$ and $S_{p}^{(m)} f$, it follows that

$$
E_{p}^{[m, \alpha]} f \sim O\left(m^{-p}\right) \text { as } m \rightarrow \infty
$$

and that $E_{p}^{[m, \alpha]} f=0$ when $\phi(x)$ is a polynomial of degree $p-2$ or less. Expansion (2.11) appears in Lyness and Ninham [4] as a special case of formula (8.1) of that paper.

In the case that $a=0$ and $b=1$, the expansion (2.11) reduces to the conventional Euler-Maclaurin summation formula. In this case definition (2.2b) rather than (2.2a) is valid.

The Möbius inversion of the Poisson summation formula now proceeds rather as in Section 6 of Part I. We may take various special cases of (2.12) and obtain different results. To obtain the result corresponding to (1.3) we set $\alpha=1$ in (2.12), which leaves

$$
E_{p}^{(m, 1)} f=2 \sum_{r=1}^{\infty} C_{p}^{(r m)} f, \quad m=1,2,3, \cdots, \quad p \geqq 2 .
$$

This may be inverted, as in Part I, Section 6 to give

$$
2 C_{p}^{(m)} f=\sum_{s=1}^{\infty} \mu_{s} E_{p}^{[m s, 1]} f, \quad p \geqq 2 .
$$

Expressed in full by means of (2.11) and (2.7) this Eq. (2.15) becomes

$$
\begin{aligned}
& 2 C^{(m)} f=-2 \sum_{a=1}^{p-1} \frac{1}{(2 \pi m)^{a}} \\
& \cdot\left\{\phi^{(a-1)}(b) \cos (2 \pi m b+\pi q / 2)-\phi^{(a-1)}(a) \cos (2 \pi m a+\pi q / 2)\right\} \\
&+ \sum_{s=1}^{\infty} \mu_{s}\left[R^{[m s, 1]} f-I f-\sum_{a=1}^{p-1} \frac{1}{(m s)^{a}}\right. \\
&\left.\left\{\frac{\bar{B}_{q}(-m s b)}{q !} \phi^{(a-1)}(b)-\frac{\bar{B}_{q}(-m s a)}{q !} \phi^{(a-1)}(a)\right\}\right], \\
& p \geqq 2 .
\end{aligned}
$$

This formula expresses the Fourier cosine coefficient of $f(x)$ as the sum of the first $p-1$ terms of its asymptotic expansion, together with an infinite sum (over index $s$ ). The elements of this sum are $\mu_{s} E_{p}^{[m s, 1]} f$. In view of the order relation (2.13) the ultimate convergence rate is as $s^{-p}$. In practice then for moderate values of $p$ the terms in this series decrease and the series may be truncated at a point where the error incurred by omitting the rest of the series is considered to be less than the required tolerance in the approximation to the Fourier cosine coefficient.

It is convenient to generalize slightly formula (2.16). We note that in order to take advantage of a rapid convergence rate it is necessary to use some moderate 
value of $p$. Thus in practice one has to evaluate-analytically or otherwise-the first $p-1$ derivatives of $\phi(x)$ at $x=a$ and at $x=b$. If these derivatives are evaluated numerically they are unlikely to be exact; in fact they may have large errors due to amplification of round-off error in function values. Consequently the formula actually used for the computation of $C^{(m)} f$ differs from (2.16) in two major respects. The sum over index $s$ will be truncated to include only terms for which $m s \leqq \bar{s}$. The quantities $\phi^{(a-1)}(b)$ and $\phi^{(a-1)}(a)$ will be replaced by approximations $\tilde{\phi}^{(a-1)}(b)$ and $\tilde{\phi}^{(a-1)}(a)$.

It turns out that Eq. (2.16) is an identity in the $2 p-4$ parameters $\phi^{(a-1)}(b)$, $\phi^{(a-1)}(a), q=2,3, \cdots, p-1$, and so remains valid whatever numbers may be used in their stead. To show this we proceed as follows. We consider the Möbius inversion of series (2.10). This may be rewritten as a set of equivalent equations as follows.

$$
\frac{\bar{B}_{q}(-m x)}{m^{q}}=-2 q ! \sum_{r=1}^{\infty} \frac{\cos (2 \pi m r x+\pi q / 2)}{(2 \pi m r)^{Q}}, \quad m=1,2,3, \cdots .
$$

These equations are in the form

$$
G(m)=\sum_{s=1}^{\infty} F(m s), \quad m=1,2,3, \cdots,
$$

with the identification

$$
G(m)=\frac{\bar{B}_{q}(-m x)}{m^{q}} ; \quad F(m)=-\frac{2 q ! \cos (2 \pi m x+\pi q / 2)}{(2 \pi m)^{q}} .
$$

When $q \geqq 2,(2.18)$ may be inverted to give

$$
F(m)=\sum_{s=1}^{\infty} \mu_{s} G(m s), \quad m=1,2,3, \cdots,
$$

which is the same as

$$
-\frac{2 \cos (2 \pi m x+\pi q / 2)}{(2 \pi m)^{q}}=\sum_{s=1}^{\infty} \frac{\mu_{s} \bar{B}_{q}(-m s x)}{(m s)^{q} q !}, \quad q \geqq 2 .
$$

We may now construct a formula analogous to (2.16). We write (2.16) for the case $p=2$. This is

$$
\begin{aligned}
2 C^{(m)} f= & \frac{-2}{2 \pi m}\{\phi(b) \cos (2 \pi m b+\pi / 2)-\phi(a) \cos (2 \pi m a+\pi / 2)\} \\
& +\sum_{s=1}^{\infty} \mu_{s}\left[R^{[m s, 1]} f-I f-\frac{1}{m s}\left\{\bar{B}_{1}(-m s b) \phi(b)-\bar{B}_{1}(-m s a) \phi(a)\right\}\right] .
\end{aligned}
$$

We take $2 p-4$ arbitrary numbers $\tilde{\phi}^{(q-1)}(b), \tilde{\phi}^{(a-1)}(a), q=2,3, \cdots, p-1$. It follows directly from (2.21) that

$$
2 \sum_{a=2}^{p-1} \frac{\tilde{\phi}^{(a-1)}(b) \cos (2 \pi m b+\pi q / 2)}{(2 \pi m)^{a}}+\sum_{s=1}^{\infty} \mu_{s} \sum_{q=2}^{p-1} \frac{\tilde{\phi}^{(a-1)}(b) \bar{B}_{q}(-m s b)}{(m s)^{a} q !}=0
$$

and a similar identity with $a$ replacing $b$ holds also. Adding these two identities into (2.22) and rearranging slightly the order of the terms gives the following equation 


$$
\begin{aligned}
& 2 C^{(m)} f=\frac{-2}{2 \pi m}\{\phi(b) \cos (2 \pi m b+\pi / 2)-\phi(a) \cos (2 \pi m a+\pi / 2)\} \\
&++\sum_{a=2}^{p-1} \frac{-2}{(2 \pi m)^{a}}\left\{\tilde{\phi}^{(a-1)}(b) \cos (2 \pi m b+\pi q / 2)-\tilde{\phi}^{(a-1)}(a) \cos (2 \pi m a+\pi q / 2)\right\} \\
&+ \sum_{s=1}^{\infty} \mu_{s}\left[R^{[m s, 1]} f-I f-\frac{1}{m s}\left\{\bar{B}_{1}(-m s b) \phi(b)-\bar{B}_{1}(-m s a) \phi(a)\right\}\right. \\
&\left.-\sum_{a=2}^{p-1} \frac{1}{(m s)^{q}}\left\{\frac{\bar{B}_{q}(-m s b)}{q !} \tilde{\phi}^{(q-1)}(b)-\frac{\bar{B}_{q}(-m s a)}{q !} \tilde{\phi}^{(q-1)}(a)\right\}\right], \\
& p>2 .
\end{aligned}
$$

This formula closely resembles Eq. (2.16). The difference is that the quantities $\phi^{(q-1)}(a)$ and $\phi^{(a-1)}(b), q \geqq 2$, have been replaced by a set of arbitrary numbers denoted, for convenience, by $\tilde{\phi}^{(a-1)}(a)$ and $\tilde{\phi}^{(a-1)}(b)$.

In applications then, approximate values of derivatives may be used in (2.16) and the formula is still valid. The effect of using approximations is described in Part I. Briefly the approximation $\widetilde{E}_{p}^{[m s, 1]} f$ to the term $E_{p}^{[m s, 1]} f$ which occurs in the square bracket in (2.16) is no longer $O\left(s^{-p}\right)$ but contains elements of order $O\left(s^{-q}\right), q=1,2 \ldots$ $p-1$. The size of such low-order elements depends on the accuracy of the approximations. These elements cause the sequence $\widetilde{E}_{p}^{[s, 1]} f$ to converge more slowly than the sequence $E_{p}^{[s, 1]} f$ and consequently a higher value of $\bar{s}$ is required to attain the same accuracy. Thus the penalty for using poor approximations for the derivatives is a longer calculation. The accuracy of the final result is not in general affected.**

Other formulas for $C^{(m)} f$ and for $S^{(m)} f$ may be obtained by inverting other forms of the Poisson summation formula (2.12). While the results can be expressed in terms of Bernoulli functions (2.10), it is less cumbersome to use Euler functions, defined by

$$
\bar{E}_{q}(x)=4 q ! \sum_{k=1 ;(k \text { odd })}^{\infty} \frac{\sin (k \pi x-\pi q / 2)}{(\pi k)^{q+1}}, \quad q=0,1,2, \cdots .
$$

These functions have period 2 and coincide with the Euler polynomials $E_{a}(x)$ in the interval $0<x<1$ and with $(-1)^{a-1} E_{a}(x)$ in the interval $-1<x<0$. Various properties of the Bernoulli and Euler polynomials are listed in Abramowitz and Stegun [1, pages 803 et seq.]. A convenient relation which is employed subsequently is

$$
\bar{E}_{n}\left(2 x+\frac{1}{2}\right)=-\frac{2^{n+1}}{n+1}\left(\bar{B}_{n+1}\left(x+\frac{1}{4}\right)-\bar{B}_{n+1}\left(x+\frac{3}{4}\right)\right) .
$$

We return now to (2.12) but instead of specifying $\alpha=1$ to obtain (2.14) we choose a linear combination as follows

$$
E_{p}^{[m, 1]} f-E_{p}^{[2 m, 1]} f=2 \sum_{r=1 ;(r \text { odd })}^{\infty} C_{p}^{(m r)} f
$$

** It is only the error (whether due to round-off or other causes) in the derivatives which is eliminated. Other round-off errors such as accumulation error naturally affect the result and would have a more pronounced effect in the longer calculation. 
Then, proceeding in the same way we find a variant of (2.24) in which the final sum over index $s$ in (2.24) is replaced by the expression

$$
\begin{aligned}
2 C_{p}^{(m)} f=\sum_{-1 ;(s \text { odd })}^{\infty} \frac{\mu_{s}}{2}[ & 2\left(R^{[m s, 1]} f-R^{[2 m s, 1]} f\right)+\frac{1}{2 m s}\{\phi(b)-\phi(a)\} \\
& +\sum_{a=2}^{p-1} \frac{1}{(2 m s)^{q}}\left\{\frac{E_{q-1}(-2 m s b)}{(q-1) !} \tilde{\phi}^{(a-1)}(b)\right. \\
& \left.\left.\quad-\frac{E_{q-1}(-2 m s a)}{(q-1) !} \tilde{\phi}^{(a-1)}(a)\right\}\right] .
\end{aligned}
$$

This formula stands in the same relation to (2.24) as does formula (6.14) of Part I to (6.11) of Part I. The similarities and differences are discussed in Part I.

A formula for $S^{(m)} f$ may be obtained from (2.12) by inverting the special case

$$
\frac{1}{2}\left(E_{p}^{[m,-1 / 2]} f-E_{p}^{(m, 1 / 2)} f\right)=2 \sum_{r=1}^{\infty}(-1)^{r-1} S_{p}^{((2 r-1) m)} f .
$$

This leads to

$$
\begin{aligned}
& 2 S^{(m)} f=\frac{-2}{2 \pi m}\{\phi(b) \sin (2 \pi m b+\pi q / 2)-\phi(a) \sin (2 \pi m a+\pi q / 2)\} \\
& +\sum_{a=2}^{p-1} \frac{-2}{(2 \pi m)^{a}}\left\{\tilde{\phi}^{(a-1)}(b) \sin (2 \pi m b+\pi q / 2)\right. \\
& \left.-\tilde{\phi}^{(a-1)}(a) \sin (2 \pi m a+\pi q / 2)\right\} \\
& +\sum_{.-1 ;(\text { odd })}^{\infty}(-1)^{(\cdot-1) / 2} \\
& \cdot \frac{\mu_{s}}{2}\left[\left(R^{[m s,-1 / 2]} f-R^{[m s, 1 / 2]}\right)+\frac{1}{2 m s}(\phi(b)-\phi(a))\right. \\
& +\sum_{q=2}^{p-1} \frac{1}{(2 m s)^{q}}\left\{\frac{E_{q-1}(-2 m s b+1 / 2)}{q-1 !} \tilde{\phi}^{(q-1)}(b)\right. \\
& \left.\left.-\frac{E_{q-1}(-2 m s a+1 / 2)}{q-1 !} \tilde{\phi}^{(a-1)}(a)\right\}\right] \text {, } \\
& p \geqq 2 .
\end{aligned}
$$

We close this section with a point of theoretical interest only. The reader will have noticed, on comparing (2.16) with (2.24), that while quantities such as $\phi^{(a-1)}(b)$, $q \geqq 2$, may be replaced by approximations $\tilde{\phi}^{(q-1)}(b)$, the possibility of a corresponding replacement of $\phi(b)$ has not been established. While not of practical significance, it would be aesthetically satisfying if such a replacement could be made. The statement of (2.24) would then be shorter.

If this were possible, Eq. (2.22) would take the form

$$
2 C^{(m)} f=\sum_{s=1}^{\infty} \mu_{s}\left[R^{[m s, 1]} f-I f\right] .
$$

Eq. (2.31) has been established (in Part I) for the case $a=0, b=1$, that is if $f(x)$ is continuous throughout the interval $[0,1]$. However the author is unable to either 
prove or disprove this result when $f(x)$ has discontinuities. The point in the above derivation at which the specialization is made is where (2.15) is derived from (2.14). Using a standard sufficient condition for Möbius inversion, the restriction $p \geqq 2$ occurs. However a set of necessary and sufficient conditions for Möbius inversion do not seem to be known.

\section{Comments on Applications.}

3(i). Piecewise Continuous Function. The extension to the case where $f(x)$ is piecewise continuous is straightforward. One need simply apply the formula (2.24) to each section in turn. A general formula is obtained by adding these separate contributions. Consider, for example, a discontinuity at $x=c, a<c<b$ with $f(x)$ coinciding with $\phi_{+}(x)$ and with $\phi_{-}(x)$ according as $x>c$ and $x<c$ in the immediate vicinity of $x=c$. Under these circumstances, the terms

$$
\begin{aligned}
-\sum_{q=1}^{p-1}\left(\phi_{-}^{(q-1)}(c)-\right. & \left.\phi_{+}^{(q-1)}(c)\right) \\
& \cdot\left\{2 \cos (2 \pi m c+\pi q / 2) /(2 \pi m)^{q}+\sum_{s=1}^{\infty} \mu_{s} \frac{\bar{B}_{q}(-m s c)}{(m s)^{q} q !}\right\}=0
\end{aligned}
$$

should be included in the right-hand side of (2.16). The additional term in the sum over index $s$ ensures that the rate of convergence of the series is $O\left(s^{-p}\right)$. We note that if in reality there were no discontinuity at $x=c$ and $\phi_{+}(x) \equiv \phi_{-}(x)$, then the additional terms simply disappear. Eq. (3.1) is an identity in $\left(\phi_{-}^{(a-1)}(c)-\phi_{+}^{(a-1)}(c)\right), q \geqq 2$, and so these terms may be replaced by $\tilde{\phi}_{-}^{(a-1)}(c)-\tilde{\phi}_{+}^{(a-1)}(c)$ in forming the corresponding adjustment to (2.24).

In practice, if the cost of the calculation depends on the number of function evaluations and derivative evaluations, it is nearly always more economical to treat each subinterval separately. This takes advantage of different values of $\bar{s}$ in different subintervals. But on the other hand a simpler code results if the problem is treated as a whole.

3(ii). Trigonometrical Integrals. The formulas of Section 2 are presented in a manner which stresses the calculation of a set of Fourier coefficients. However, since $f(x)$ may be discontinuous they may clearly be applied to calculate an individual trigonometric integral, of the form

$$
\int_{\Delta}^{B} \theta(x) \cos K x d x
$$

where $\theta(x) \in C^{(p)}[A, B]$ and $K$ is not restricted to be a multiple of $2 \pi(B-A)$. This may be expressed as a Fourier coefficient in many ways, one of which is to make the analytic substitution

$$
x^{\prime}=(K x-2 \pi l) / 2 \pi m
$$

where $m$ is a positive integer and $l$ is an integer.

The integral (3.2) may then be expressed in the form

$$
\frac{2 \pi m}{K} \int_{a}^{b} \theta\left(\frac{2 \pi m x+2 \pi l}{K}\right) \cos 2 \pi m x d x
$$

where

$$
b=(K B-2 \pi l) / 2 \pi m, \quad a=(K A-2 \pi l) / 2 \pi m .
$$


If $m$ and $l$ are chosen so that

$$
0 \leqq a<b \leqq 1,
$$

the integral (3.4) may be calculated using formula (2.24).

The substitution (3.3) corresponds to scaling and it is of some interest to inquire as to the most suitable choice from the infinite range of possible values of $m$ and of $l$. A short calculation reveals that the particular choice is immaterial so far as the calculation of (3.2) is concerned. Application of (2.16) to the integral in (3.4) yields $2 \int_{A}^{B} \theta(x) \cos K x d x$

$$
\begin{aligned}
&=\sum_{q=1}^{p-1} \frac{-2}{K^{q}}\left\{\theta^{(a-1)}(B) \cos (K B+\pi q / 2)-\theta^{(q-1)}(A) \cos (K A+\pi q / 2)\right\} \\
&+\sum_{s=1}^{\infty} \mu_{s} {\left[\sum_{K A s \leq \geq \pi j \leqq K B s}^{*} \frac{2}{K S} \theta\left(\frac{2 \pi j}{K S}\right)-\int_{A}^{B} \theta(x) d x\right.} \\
&\left.\quad-\sum_{q=1}^{p-1}\left(\frac{2 \pi}{K s}\right)^{q}\left\{\frac{\bar{B}_{q}(-K s B / 2 \pi)}{q !} \theta^{(q-1)}(B)-\frac{\bar{B}_{q}(-K S A / 2 \pi)}{q !} \theta^{(q-1)}(A)\right\}\right] .
\end{aligned}
$$

The sum over index $j$ is a scaled version of the trapezoidal rule. It includes the sum of the function evaluations at all the net points $2 \pi j / K s$ which lie in the interval $(A, B)$. The asterisk indicates that if a net point coincides with either $A$ or $B$, a weighting coefficient $\frac{1}{2}$ has to be assigned to the function value $\theta(A)$ or $\theta(B)$.

Formula (3.7) is independent of the choice of $m$ or of $l$ made in its derivation. It is an identity in the $2 p-4$ quantities $\theta^{(a-1)}(B), \theta^{(a-1)}(A), q \geqq 2$.

A formula analogous to (3.7) may be derived from (2.28) and a corresponding formula for $\int_{A}^{B} \theta(x) \sin K x d x$ may be derived from (2.30) using the same substitution (3.3). These formulas do not involve the term $\int_{A}^{B} \theta(x) d x$, but require about twice the number of function values needed by (3.7) to attain comparable numerical accuracy.

3(iii). Point of Discontinuity. Formula (2.16) is interesting as an example of a calculation in which it is important to treat a discontinuity carefully. In definition (2.2a) we note that

$$
f(x)=\phi(x), \quad a<x<b,
$$

but

$$
f(a)=(1 / 2) \phi(a) ; \quad f(b)=(1 / 2) \phi(b) .
$$

There is a tendency to regard (3.9) of significance perhaps in presenting a wellrounded theory but of no practical significance. When $a$ is rational, the quantities $f(a)$ and $\phi(a)$ occur and are different. As an example, suppose $a=1 / 3$ and $b=3 / 4$. Then

$$
\begin{aligned}
& R^{[1,1]} f=0, \\
& R^{[2,1]} f=(1 / 2) \phi(1 / 2), \\
& R^{[3,1]} f=(1 / 3)((1 / 2) \phi(1 / 3)+\phi(2 / 3)), \\
& R^{[4,1]} f=(1 / 4)(\phi(1 / 2)+(1 / 2) \phi(3 / 4)) .
\end{aligned}
$$

The factor of $1 / 2$ attached to $\phi(1 / 3)$ is as important as, for example, the factor $1 / 2$ 
attached to the end values $f(0)$ and $f(1)$ in the trapezoidal rule sum. Careless coding of this calculation could lead to $f(1 / 3)$ being replaced either by $f(1 / 3-\epsilon)=0$ or by $f(1 / 3+\epsilon)=\phi(1 / 3+\epsilon)$, neither of which approximates $f(1 / 3)=(1 / 2) \phi(1 / 3)$ at all accurately. The effect of this error would be analogous to the effect of using one grossly incorrect function value in a sequence of applications of a numerical quadrature rule. While in many cases the sequence would converge, the rate of convergence might be unduly slow.

3(iv). Numerical Example. As an example we have treated the function

$$
\begin{aligned}
& f(x)=\phi(x)=e^{x}, \quad a<x<b, \\
& f(x)=(1 / 2) \phi(x), \quad x=a, \quad x=b, \\
& f(x)=0, \quad 0<x<a, \quad b<x \leqq 1 \text {, }
\end{aligned}
$$

with

$$
a=\sqrt{ } 2-1.2 \simeq 0.214, \quad b=\sqrt{ } 3-1.0 \simeq 0.732 .
$$

Analytic formulas for the derivatives $\phi^{(a)}(b), \phi^{(a)}(a)$ and for $1 f$ were employed to calculate an array of values of $E_{p}^{[m, 1]} f$ (from (2.11)) using a computer whose machine accuracy parameter is $\epsilon_{M}=3 \times 10^{-11}$. Part of this array is shown in Table 1 .

$$
\begin{aligned}
& \mathrm{s} \quad \mathrm{E}_{1}^{[\mathrm{s}, 1]} \mathrm{f} \quad \mathrm{E}_{2}^{[\mathrm{s}, 1]} \mathrm{f} \quad \mathrm{E}_{3}^{[\mathrm{s}, 1]} \mathrm{f} \quad \mathrm{E}_{4}^{[\mathrm{s}, 1]} \mathrm{f} \quad \mathrm{E}_{5}^{(\mathrm{s}, 1]} \mathrm{f} \\
& 1 \quad-8.4045336-001 \quad-3.8835404-003 \quad 2.5743991-002 \quad 3.6809058-005 \quad-6.6681135-004
\end{aligned}
$$

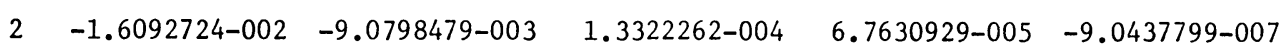

$$
\begin{aligned}
& 3 \quad 2.7399546-001 \quad 4.4893578-003 \quad-8.8436749-004 \quad-1.8911164-005 \quad 2.5181539-006 \\
& 4 \quad-1.0726669-001 \quad 4.8028584-003 \quad 7.2357935-006 \quad-6.0732187-006 \quad 1.7044763-008 \\
& 5 \quad-1.7766466-001 \quad-4.7403951-003 \quad 1.5103405-004 \quad 4.5947104-006 \quad-1.5299088-007 \\
& 6 \quad-8.4420696-003 \quad-1.4291938-003 \quad 1.9012072-006 \quad 1.2435545-006 \quad 6.2426173-010
\end{aligned}
$$

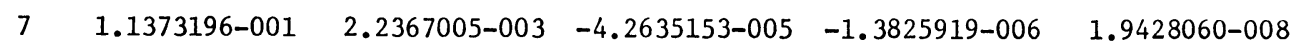

$$
\begin{aligned}
& \begin{array}{llllll}
8 & -5.8455352-002 & 1.0858591-003 & 1.1562099-005 & -4.9420469-007 & -2.9363430-009
\end{array} \\
& 9 \quad 3.6738622-002 \quad-1.7296424-003 \quad 2.6369996-006 \quad 5.5965599-007 \quad-1.7039205-009
\end{aligned}
$$

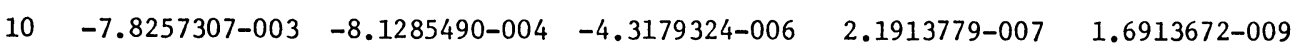

$$
\begin{aligned}
& 20 \quad-2.8129595-002 \quad-1.0538522-004 \quad 2.5465063-006 \quad 7.9220986-009 \quad-1.0029742-010 \\
& 40 \quad 1.3460510-002 \quad 6.8725215-006 \quad-2.9345814-007 \quad-2.1711545-010 \quad 6.4543201-011 \\
& 80 \quad-7.3028107-003 \quad-1.7524551-005 \quad 2.8112065-008 \quad 1.3616524-010 \quad 5.4402890-011 \\
& \text { Values of } E_{p}^{[s, 1]} f \text { calculated using (2.11) with } f(x) \text { defined by (3.11) and (3.12). }
\end{aligned}
$$


Attention is drawn to the erratic nature of the sequence for fixed $p$. While conforming to the order relation $E_{p}^{[m, 1]} f \sim O\left(m^{-p}\right)$, for fixed $p$ this function is by no stretch of the imagination a smooth function of $m$ even in this very amenable example. In practice this means that a very cautious practical convergence criterion should be applied. One might demand that

$$
\left|E_{p}^{(s+r)} f\right|(s+r)^{p}<\epsilon s^{p}, \quad r=0,1,2,3,4,
$$

before accepting $s+4=\bar{s}$ as a reasonable termination value.

4. Functions with Complex Poles near the Interval $(0,1)$. The technique of 'subtracting out the singularity' is well known in the practice of numerical quadrature. In cases in which the integrand $f(x)$ or its derivatives have singularities of a specified nature either on the interval of integration, or in the complex plane near this interval, the skillful application of this technique can lead to a significantly shorter numerical calculation at the expense of a relatively insignificant amount of analytical work. Essentially one sets

$$
f(x)=\phi(x)+r(x)
$$

and approximates If by $I \phi+R r$ instead of by $R f$. One has to choose $\phi(x)$ and $r(x)$ in such a way that $I \phi$ is known in analytic form and $r(x)$ is more amenable to the numerical quadrature technique than is $f(x)$.

In the analogous problem of calculating Fourier coefficients one may in principle apply the same technique. In practice it is more difficult. In the quadrature problem one has to choose a function $\phi(x)$ having a specified singularity for which the value of

$$
I \phi=\int_{0}^{1} \phi(x) d x
$$

is known. In the Fourier coefficient problem one has to choose $\phi(x)$ so that, in addition an analytic expression for

$$
C^{(m)} \phi=\int_{0}^{1} \phi(x) \cos 2 \pi m x d x, \quad m=1,2,3, \cdots,
$$

is known. It seems that there are relatively few such functions and those that do exist are not well known.

In this section and the next we treat one rather special class of problems in which we are able to subtract out a singularity, though the subtraction process is carried out implicitly. This class is rather small, consisting of analytic functions having complex poles near the interval of integration. Specifically, $f(x)$ is of the form

$$
f(x)=\theta(x) /\left(x^{2}-2 \lambda x+\lambda^{2}+\mu^{2}\right)^{w},
$$

where $w$ is a small positive integer and $\theta(x)$ is analytic in a convex region which contains the interval $[0,1]$ and the poles. These are of order $w$ and are located at $z=c$ and $z=\bar{c}$, where

$$
c=\lambda+i \mu, \quad \mu>0 .
$$

An additional restriction of the technique to be described is that it is more efficient 
than the standard technique only if the poles lie nearer to the interval of integration than to either of the lines $\operatorname{Re}(z)=0$ and $\operatorname{Re}(z)=1$. Specifically

$$
0<\lambda<1 ; \quad \mu / \lambda<1 ; \quad \mu /(1-\lambda)<1 .
$$

However, it may be applied in cases in which $f(x)$ has several poles satisfying this condition. These poles may be of different orders, but, corresponding to each pole, the coefficients $a_{-1}, a_{-2}, \cdots, a_{-w}$ which occur in the infinite part of the Laurent expansion

$$
f(z)=\sum_{r=-w}^{\infty} a_{r}(z-c)^{r}
$$

have to be known.

Before describing a specific technique for evaluating these Fourier coefficients, it is instructive to consider for a moment what other methods are presently available. We discuss first the simplest case, namely

$$
f(x)=1 /\left(x^{2}-2 \lambda x+\lambda^{2}+\mu^{2}\right) \text {. }
$$

Reference to Abramowitz and Stegun [1, p. 230, Formula 5.1.41] leads to the analytic result

$$
\begin{aligned}
C^{(m)} f+i S^{(m)} f=\frac{i}{2 \mu}\left[e^{2 \pi i m c}\left(E_{1}(2 \pi m(i c-i))-E_{1}(2 \pi m i c)\right)\right. & \\
& \left.-e^{2 \pi i m \bar{c}}\left(E_{1}(2 \pi m(i \bar{c}-i))-E_{1}(2 \pi m i \bar{c})\right)\right]
\end{aligned}
$$

where $E_{1}(z)$ is the exponential integral function. Standard routines for this function with complex argument are not readily available. Indeed a brief investigation into the problem of writing such a routine leads directly back to a problem very close to the one we started with.

Perhaps the easiest method known to the author for this particular example is that described in Smith and Lyness [5, pp. 246 et seq.]. Essentially, the integral is replaced by two contour integrals, which are evaluated numerically using the GaussLaguerre formula. In this case that method and the one to be described are of comparable efficiency.

When one generalizes the problem a little, it is found that the contour integration method becomes successively more difficult to apply as $\theta(x)$ becomes more sophisticated. This is because the choice of contour depends on the behavior of $f(z)$ for large $|z|$.

While the class of problems for which the following method is suitable is relatively small, at least in dealing with these problems the method is efficient.

5. Functions with Complex Poles Near the Interval $(0,1)$ : Theory. The derivation of the formulas given in Part I and in Section 2 of this paper follow a standard pattern. This involves establishing a Fourier Coefficient Asymptotic Expansion (FCAE), and using the Poisson summation formula to derive a corresponding EulerMaclaurin asymptotic expansion. The relation between the remainder terms of these respective expansions is then inverted.

In reference [3] a pair of expansions of this type was derived. These were convenient when $f(z)$ is analytic, except for some specified poles within the rectangle 
having the interval $[0,1]$ as base and height $L^{\prime}$. For convenience we suppose that there is one such pole, situated at $c=\lambda+i \mu$. A quantity which occurs in the FCAE is the residue of the function $f(z) e^{i k z}$ at $z=c$. In terms of the coefficients of the Laurent expansion (4.7) above, this is given by

$$
\operatorname{Res}(k)=e^{i k c} \sum_{r=1}^{w} a_{-r}(i k)^{r-1} /(r-1) !,
$$

and an alternate form of the FCAE (see [3, Eq. (4.19)]) is

$$
2 C^{(m)} f=\sum_{q=1}^{n} \frac{K_{2 q}}{m^{2 q}}+\operatorname{Re}(4 \pi i \operatorname{Res}(2 \pi m))+2 C_{p}^{(m)}\left(L^{\prime}\right) f
$$

where $n$ is the integer part of $(p-1) / 2$. This differs from the standard form in that the standard remainder term, denoted by $2 C_{p}^{(m)} f$, has been split into two parts, i.e.,

$$
2 C_{p}^{(m)} f=\operatorname{Re}(4 \pi i \operatorname{Res}(2 \pi m))+2 C_{p}^{(m)}\left(L^{\prime}\right) f .
$$

Both $C_{p}^{(m)} f$ and $C_{p}^{(m)}\left(L^{\prime}\right) f$ are of the same order $O\left(m^{-p}\right)$. But for moderate values of $m$ the numerically dominant term in $C_{p}^{(m)} f$ is of order $O\left(m^{-1} e^{-2 \pi m \mu}\right)$, while in $C_{p}^{(m)}\left(L^{\prime}\right) f$ the numerically dominant term is of order $O\left(m^{-1} e^{-2 \pi m L^{\prime}}\right)$, where $L^{\prime}>\mu$. Thus, while $C_{p}^{(m)} f$ and $C_{p}^{(m)}\left(L^{\prime}\right) f$ are of the same order, for moderate values of $m$ the remainder term $C_{p}^{(m)}\left(L^{\prime}\right) f$ may be much smaller in magnitude than $C_{p}^{(m)} f$.

The derivation of the Euler-Maclaurin expansion which corresponds to (5.2) is carried out in detail in reference [3]. The result is

$$
R^{[m, \alpha]} f-I f=\sum_{q=1}^{p-1} \frac{\bar{B}_{q}\left(t_{\alpha}\right)}{q !} \frac{f^{(q-1)}(1)-f^{(q-1)}(0)}{m^{q}}+\Delta^{[m, \alpha]}+E_{p}^{[m, \alpha]}\left(L^{\prime}\right) f .
$$

Here the term arising from the residue term in (5.2) is

$$
\Delta^{[m, \alpha]}=\operatorname{Re} \sum_{r=1}^{\infty} e^{-2 \pi i r t \alpha} 4 \pi i \operatorname{Res}(2 \pi m r)
$$

and the remainder term in (5.4) is related to that in (5.2) by

$$
E_{p}^{[m, \alpha]}\left(L^{\prime}\right) f=2 \sum_{r=1}^{\infty} \cos 2 \pi r t_{\alpha} C_{p}^{(r m)}\left(L^{\prime}\right) f+2 \sum_{r=1}^{\infty} \sin 2 \pi r t_{\alpha} S_{p}^{(r m)}\left(L^{\prime}\right) f .
$$

We now proceed to invert a special case of this formula. If we set $\alpha=1$ we have

$$
E_{p}^{[m, 1]}\left(L^{\prime}\right) f=2 \sum_{r=1}^{\infty} C_{p}^{(m r)}\left(L^{\prime}\right) f, \quad m=1,2,3, \cdots .
$$

This may be inverted to give

$$
2 C_{p}^{(m)}\left(L^{\prime}\right) f=\sum_{s=1}^{\infty} \mu_{s} E_{p}^{[m s, 1]}\left(L^{\prime}\right) f, \quad p>1 .
$$

Expressed in full by means of (5.2) and (5.4), this is

$$
\begin{aligned}
2 C^{(m)} f= & \sum_{a=1}^{n} \frac{K_{2 q}}{m^{2 a}}+\operatorname{Re}(4 \pi i \operatorname{Res}(2 \pi m)) \\
& +\sum_{s=1}^{\infty} \mu_{s}\left[R^{[m s, 1]} f-I f-\sum_{a=1}^{n} \frac{K_{2 q} \zeta(2 q)}{(m s)^{2 Q}}-\Delta^{[m s, 1]}\right] .
\end{aligned}
$$


In numerical applications, the residue term occurs in the form

$$
\operatorname{Re}(4 \pi i \operatorname{Res}(2 \pi m))=\operatorname{Re}\left\{4 \pi i e^{2 \pi i m(\lambda+i \mu)} \sum_{s=0}^{w-1} a_{-s-1}(2 \pi i m)^{s} / s !\right\}
$$

The term $\Delta^{[m, \alpha]}$ given by (5.5) may be expressed in terms of these parameters by carrying out the sum over index $r$ analytically. Simple expressions for these sums are available. Further details are given in reference [3]. The result is

$$
\Delta^{[m, \alpha]}=\operatorname{Re}\left\{4 \pi i \sum_{s=0}^{w-1} a_{-s-1} m^{s} \psi_{s}\left(t_{\alpha}, m c\right) / s !\right\} .
$$

Here the functions $\psi_{\diamond}\left(t_{\alpha}, m c\right)$ are defined in reference [3]. Examples are given in (6.8) and (6.13) below.

We emphasize here that the calculation which leads from (5.10) to (5.11) by means of (5.5) is algebraic in nature. So far as that calculation is concerned, the parameters $c=\lambda+i \mu$ as long as $\mu>0$, and $a_{-r}, r=1,2, \cdots, w$, need not correspond to any actual function. These parameters are incidental. In fact they appear in formula (5.9) on much the same footing as do the parameters $K_{2 q}$.

We now show that formula (5.9) is actually an identity in these parameters. If these parameters are inaccurately or incorrectly calculated (with the single restriction that $\mu>0$ ) formula (5.9) still holds. We have to prove Theorem 5.17 below, which is an example of Möbius inversion. Before doing this we prove two lemmas

Lemma 1. Given any positive $\alpha$, there exists a number $A$ such that

$$
|\operatorname{Res}(k)|<A e^{-\alpha k / 2 \pi}, \quad k>0 .
$$

Proof. A polynomial $P_{w}(z)$ of degree $w$ is an entire function of any positive order. Thus, given any positive $B$ there exists a number $A$ such that $\left|P_{w}(z)\right|<A e^{B|z|}$ for all $z$. Applying this inequality with $B<\mu$ in (5.1) and setting $\alpha=2 \pi(\mu-B)$ leads directly to (5.12).

LEMMA 2. Given any positive $\alpha$, there exists a number $A$ such that

$$
L=\sum_{l=1}^{\infty} \sum_{k=1}^{\infty}|\operatorname{Re}(4 \pi i \operatorname{Res}(2 \pi k l m))|<\frac{4 \pi A e^{-\alpha m}}{\left(1-e^{-\alpha m}\right)^{2}}, \quad m=1,2, \cdots .
$$

Proof. This follows using standard inequalities, the result of Lemma 1, and the formula for the sum of a geometric progression twice. Thus,

$$
\begin{aligned}
L & <\sum_{l=1}^{\infty} \sum_{k=1}^{\infty} 4 \pi A e^{-\alpha k l m}=4 \pi A \sum_{l=1}^{\infty} e^{-\alpha l m} /\left(1-e^{-\alpha l m}\right) \\
& <\frac{4 \pi A}{1-e^{-\alpha m}} \sum_{l=1}^{\infty} e^{-\alpha l m}=\frac{4 \pi A e^{-\alpha m}}{\left(1-e^{-\alpha m}\right)^{2}} .
\end{aligned}
$$

We are now in a position to carry out the Möbius inversion. We have by definition

$$
\Delta^{(m, 1)}=\operatorname{Re} \sum_{s=1}^{\infty} 4 \pi i \operatorname{Res}(2 \pi m s), \quad m=1,2,3, \cdots .
$$

This is of the form $G(m)=\sum_{s-1}^{\infty} F(m s)$ and may be formally inverted to give $F(m)=$ $\sum_{s=1}^{\infty} \mu_{s} G(m s)$ so long as

$$
\sum_{l=1}^{\infty} \sum_{k=1}^{\infty}\left|\mu_{k} F(k l m)\right|<\infty, \quad m=1,2, \cdots .
$$


Since $\left|\mu_{k}\right| \leqq 1$, this condition is established by Lemma 2 . Consequently, we have

THEOREM 5.17. If $\operatorname{Res}(k)$ is the function of $c=\lambda+i \mu$ and of $a_{-1}, a_{-2}, \cdots, a_{-w}$ given by (5.1) and $\Delta^{[m, 1]}$ is the function of the same variables defined by (5.11) or by (5.5), and $\mu>0$, then

$$
\operatorname{Re}(4 \pi i \operatorname{Res}(2 \pi m))=\sum_{s=1}^{\infty} \mu_{s} \Delta^{[m s, 1]}, \quad m=1,2, \cdots .
$$

Consequently Eq. (5.9), besides being an identity in the quantities $K_{2 a}$, is also an identity in the parameters which occur in Res $(k)$ and also in $\Delta^{[m, 1]}$.

The formulas which correspond to the other representations of Part I are derived in much the same way. These have very much the same properties. Thus,

$$
\begin{aligned}
2 C^{(m)} f & =\sum_{a=1}^{n} \frac{K_{2 q}}{m^{2 q}}+\operatorname{Re}(4 \pi i \operatorname{Res}(2 \pi m)) \\
+ & \sum_{\cdot-1 ;(s \text { odd })}^{\infty} \mu_{s}\left[R^{[m s, 1]} f-R^{[2 m s, 1]} f-\sum_{a=1}^{n} \frac{K_{2 q} \lambda(2 q)}{(m s)^{2 q}}-\Delta^{[m s, 1]}+\Delta^{[2 m s, 1]}\right]
\end{aligned}
$$

and

$$
\begin{aligned}
2 S^{(m)} f=\sum_{q=1}^{n} \frac{K_{2 q-1}}{m^{2 q-1}}+\operatorname{Im}(4 \pi i \operatorname{Res}(2 \pi m)) & \begin{array}{r}
+\sum_{s=1 ;(s \text { odd })}^{\infty}(-1)^{(s-1) / 2} \mu_{s}\left[\frac{1}{2}\left(R^{[m s,-1 / 2]} f-R^{[m s,+1 / 2]} f\right)-\sum_{q=1}^{n} \frac{K_{2 q-1} \beta(2 q-1)}{(m s)^{2 q-1}}\right. \\
\left.-\frac{1}{2}\left(\Delta^{[m s,-1 / 2]}+\Delta^{[m s, 1 / 2]}\right)\right]
\end{array}
\end{aligned}
$$

These correspond to Eqs. (6.14) and (6.15) of Part I. The quantities $K_{2 q-1}, \lambda(q)$ and $\beta(q)$ are defined in (6.16) and (5.16) of Part I.

6. Functions with Complex Poles near the Interval $(0,1)$ : Applications. In this section we discuss briefly some of the points which arise in the practical applications of these formulas. The discussion is limited to a method based on Eq. (5.9) which we term 'the modified method' and this is compared with a method based on Eq. (1.3) termed 'the standard method.' However, the discussion is of equal relevance in the context of the variant methods based on Eqs. (5.18) and (5.19).

The advantage of using the modified method (5.9) rather than the standard method is simply computational. If $\mu$ is small, the set of quantities $E_{2 n+2}^{[s, 1]} f, s=1$, $2,3, \cdots$, take on large values for moderate $s$ and a large value of $\bar{s}$ may be required before the user is confident that

$$
\sum_{s=s+1}^{\infty}\left|E_{2 n+2}^{[s, 1]} f\right|<2 \epsilon
$$

On the other hand the size of the quantities $E_{2 n+2}^{[s, 1]}\left(L^{\prime}\right) f, s=1,2,3, \cdots$, is generally smaller for moderate $s$ and so a smaller value of $\bar{s}$, denoted by $\bar{s}\left(L^{\prime}\right)$, may be required before the user is confident that

$$
\sum_{s=s\left(L^{\prime}\right)+1}^{\infty}\left|E_{2 n+2}^{[s, 1]}\left(L^{\prime}\right) f\right|<2 \epsilon
$$


If this happens the effect of using the modified method is that the user is saved the calculation of

$$
R^{[\bullet, 1]} f, \quad s=\bar{s}\left(L^{\prime}\right)+1, \cdots, s,
$$

but the additional cost incurred includes the calculation of the coefficients in Res $(k)$ and the calculation of

$$
\Delta^{[0,1]}, \quad s=1,2, \cdots, s\left(L^{\prime}\right)
$$

In the case of a pole of order 1 , this additional work is almost trivial. If $f(x)$ is given in the form

$$
f(x)=\theta(x) /(x-c)(x-\bar{c})
$$

we find

$$
a_{-1}=\theta(c) / 2 i \mu
$$

and the terms occurring in (5.9) are given by

$$
\begin{gathered}
\operatorname{Re}(4 \pi i \operatorname{Res}(2 \pi m))=\operatorname{Re}\left(4 \pi i a_{-1} e^{2 \pi i m c}\right), \\
\Delta^{[m, 1]}=\operatorname{Re}\left(4 \pi i a_{-1} /\left(e^{-2 \pi i m c}-1\right)\right) .
\end{gathered}
$$

Since values of these quantities are required successively for $m=1,2, \cdots$, the author has found it convenient to calculate these using complex arithmetic, updating the exponential using complex multiplication.

In the case of poles of higher order, the additional analytic work becomes more significant. Thus, if $f(x)$ has a pole of order 2 and takes the form

$$
f(x)=\theta(x) /\{(x-c)(x-\bar{c})\}^{2}
$$

we find

$$
\begin{aligned}
& a_{-1}=\left(\theta(c)-i \mu \theta^{\prime}(c)\right) / 4 i \mu^{3}, \\
& a_{-2}=-\theta(c) / 4 \mu^{2},
\end{aligned}
$$

and, after expressing $\psi_{1}(1, m c)$ in analytic form

$$
\begin{aligned}
& \operatorname{Re}(4 \pi i \operatorname{Res}(2 \pi m))=\operatorname{Re}\left(4 \pi i e^{2 \pi i m c}\left(a_{-1}+i k a_{-2}\right)\right), \\
& \Delta^{[m, 1]}=\operatorname{Re}\left(4 \pi i\left\{\frac{a_{-1}}{e^{-2 \pi i m c}-1}-\frac{2 \pi i m a_{-2} e^{-2 \pi i m c}}{\left(e^{-2 \pi i m c}-1\right)^{2}}\right\}\right) .
\end{aligned}
$$

Besides the human effort required to obtain these formulas, there is the added complication that the value of-or an approximation to- $\theta^{\prime}(c)$ is also needed.

In Tables $2 \mathrm{a}$ and $2 \mathrm{~b}$ the behavior of the sequences $E_{2 n+2}^{(s)} f$ and $E_{2 n+2}^{(s)}\left(L^{\prime}\right) f$ is illustrated. These sequences have been calculated for the function

$$
f(x)=\frac{1}{x^{2}-2 \lambda x+\lambda^{2}+\mu^{2}}, \quad \lambda=0.4, \quad \mu=0.1,
$$

using a computer whose machine accuracy parameter $\epsilon_{M}$ is $3 \times 10^{-11}$. Analytic expressions for the values of If and the derivatives $f^{(2 q-1)}(1)-f^{(2 q-1)}(0)$ and in Table $2 \mathrm{~b}$ the residue $a_{-1}$ were used. 


\begin{tabular}{|c|c|c|c|c|c|}
\hline & & & TABLE $2 \mathrm{a}$ & & \\
\hline $\mathrm{s}$ & $E_{2}^{[s, 1]} f$ & $E_{4}^{[s, 1]} f$ & $E_{6}^{[s, 1]} f$ & $\mathrm{E}_{8}^{[\mathrm{s}, 1]} \mathrm{f}$ & $\mathrm{E}_{10}^{[s, 1]} \mathrm{f}$ \\
\hline 1 & $-2.3022125+001$ & $-1.9984860+001$ & $-2.2752 .968+001$ & $-1.4061678+001$ & $-6.2375858+001$ \\
\hline 2 & $-1.6838922-001$ & $5.9092710-001$ & $4.1792036-001$ & $5.5372177-001$ & $3.6499451-001$ \\
\hline 3 & $1.3027016+000$ & $1.6401755+000$ & $1.6060013+000$ & $1.6179236+000$ & $1.6105597+000$ \\
\hline 4 & $-4.1624210+000$ & $-3.9725919+000$ & $-3.9834049+000$ & $-3.9812830+000$ & $-3.9820202+000$ \\
\hline 5 & $2.7203230+000$ & $2.8418136+000$ & $2.8373847+000$ & $2.8379409+000$ & $2.8378172+000$ \\
\hline 6 & $-1.2437389+000$ & $-1.1593704+000$ & $-1.1615063+000$ & $-1.1613200+000$ & $-1.1613483 \div 000$ \\
\hline 7 & $1.7011851-001$ & $2.3210352-001$ & $2.3095062-001$ & $2.3102450-001$ & $2.3101612-001$ \\
\hline 8 & $7.8382467-002$ & $1.2583974-001$ & $1.2516393-001$ & $1.2519708-001$ & $1.2519420-\mathrm{CO}$ \\
\hline 9 & $-2.1478625-001$ & $-1.7728915-001$ & $-1.7771106-001$ & $-1.7769470-001$ & $-1.7769582-001$ \\
\hline & $8.7450332-002$ & $1.1782298-001$ & $1.1754617-001$ & $1.1755486-001$ & $1.1755438-001$ \\
\hline 0 & $-7.3568779-003$ & $2.3628526-004$ & $2.1898458-004$ & $2.1912039-004$ & $2.1911850-004$ \\
\hline 0 & $-1.8972093-003$ & $1.0814667-006$ & $1.7456600-010$ & $2.2964630-009$ & $2.2890909-009$ \\
\hline & $-4.7450373-004$ & $6.8968163-008$ & $1.3874057-009$ & $1.4205604-009$ & $1.4205316-009$ \\
\hline
\end{tabular}

The actual value of $C^{(1)} f$ is about 20.1. The magnitude of subsequent cosine Fourier coefficients varies with increasing $m$ roughly as $m^{-2}$. For example, $C^{(10)} f \simeq$ $-9.1 \times 10^{-3}$ and $C^{(1024)} f \simeq-8.8 \times 10^{-7}$.

In a Fourier coefficient calculation, the computer would calculate various elements of one of these tables, terminating when a practical convergence criterion was satisfied. If the required accuracy were $\epsilon=0.5 \times 10^{-7}$, the terminal values using the standard method (Table 2a) might be $\bar{s}=33$ while, using the method based on Eq. (5.8) (Table 2b) this might be $\bar{s}\left(L^{\prime}\right)=10$. The number of function values required are 345 and 33 respectively. The evaluation of the actual Fourier coefficients is at this stage a matter of substitution in a simple formula.

It is very easy to invent examples with more flamboyant differences. If one alters $\mu$ to 0.01 (a peak of height 1000) in the example given, the author's standard routine abandons the problem with $\bar{s}=100$, the number of function values 3045 and present accuracy about 1.0. The modified method however behaves in much the same way as illustrated in Table $2 b$. The Fourier coefficients are now about ten times as large, but the same value $\bar{s}\left(L^{\prime}\right)=10$ is required to attain the same accuracy $\epsilon=0.5 \times 10^{-7}$. 
TABLE $2 b$

\begin{tabular}{|c|c|c|c|c|c|}
\hline & $E_{2}^{[s, l]}\left(L^{\prime}\right) f$ & $E_{4}^{[s, 1]}\left(L^{\prime}\right) f$ & $E_{6}^{[s, 1]}\left(L^{\prime}\right) f$ & $E_{8}^{[s, 1]}\left(L^{\prime}\right) f$ & $E_{10}^{[s, 1]}\left(L^{\prime}\right) f$ \\
\hline 1 & $-2.0701780+000$ & $9.6708723-001$ & $-1.8010206+000$ & $6.8902697+000$ & $-4.1423911+001$ \\
\hline 2 & $-6.5061804-001$ & $1.0869828-001$ & $-6.4308461-002$ & $7.1492950-002$ & $-1.1723432-001$ \\
\hline 3 & $-3.1105746-001$ & $2.6416459-002$ & $-7.7577115-003$ & $4.1644974-003$ & $-3.1993466-003$ \\
\hline 4 & $-1.8062783-001$ & $9.2012461-003$ & $-1.6116750-003$ & $5.1022205-004$ & $-2.2699387-004$ \\
\hline 5 & $-1.1752194-001$ & $3.9686712-003$ & $-4.6030129-004$ & $9.5941301-005$ & $-2.7742994-005$ \\
\hline 6 & $-8.2394956-002$ & $1.9735240-003$ & $-1.6236166-004$ & $2.3922854-005$ & $-4.8421498-006$ \\
\hline 7 & $-6.0898689-002$ & $1.0863164-003$ & $-6.6581462-005$ & $7.2932853-006$ & $-1.0876065-006$ \\
\hline 8 & $-4.6812032-002$ & $6.4523816-004$ & $-3.0569408-005$ & $2.5852314-006$ & $-2.9451985-007$ \\
\hline 9 & $-3.7090522-002$ & $4.0657955-004$ & $-1.5323792-005$ & $1.0304029-006$ & $-9 \cdot 1964466-008$ \\
\hline 0 & $-3.0104082-002$ & $2.6857076-004$ & $-8.2400220-006$ & $4.5126762-007$ & $-3.1874151-008$ \\
\hline 20 & $-7.5759949-003$ & $1.7168312-005$ & $-1.3236222-007$ & $3.4391903-009$ & $1.5519177-009$ \\
\hline 40 & $-1.8972101-003$ & $1.0807025-006$ & $-5.8956714-010$ & $1.5323299-009$ & $1.5249577-009$ \\
\hline 80 & $-4.7450373-004$ & $6.8968163-008$ & $1.3874057-009$ & $1.4205604-009$ & $1.4205316-009$ \\
\hline
\end{tabular}

Tables $2 \mathrm{a}$ and $2 \mathrm{~b}$ illustrate the spirit of the modification and to some extent the spirit of the MIPS method. Comparison of the two tables shows that $E_{2 n+2}^{(s)} f$ and $E_{2 n+2}^{(s)}\left(L^{\prime}\right) f$ are virtually identical for large $s$, but are significantly different for small $s$. Because the calculation actually takes place using small values of $s$, considerable advantage accrues from using the modified method. This is despite the fact that asymptotically the formulas are identical.

Another point of interest is that it is possible to switch from the standard method to the modified method in the middle of a calculation. This is because

$$
E_{2 n+2}^{[m, 1]}\left(L^{\prime}\right) f=E_{2 n+2}^{[m, 1]} f-\Delta^{[m, 1]} \text {. }
$$

This is illustrated in the Tables $2 a$ and $2 b$. In a hand calculation of Table $2 a$ it might be noticed that for $s=6,7,8,9$ the values of $E_{2 n}^{[s, 1]} f$ are virtually independent of $n$ for $n=2,3,4,5$ and are growing smaller with increasing $s$ very slowly. At this stage the user could calculate the set of numbers $\Delta^{[s, 1]}$ and update Table 2a using (6.15) to form Table $2 b$.

In practice, calculations which include this amount of supervision are rare. 
But the remarks are equally valid in the context of updating an automatic code. The additional coding needed to modify a running code in this way is not significant.

Argonne National Laboratory

Argonne, Illinois 60439

1. M. Abramowitz \& I. A. Stegun, (Editors), Handbook of Mathematical Functions with Formulas, Graphs, and Mathematical Tables, Nat. Bur. Standards Appl. Math. Series, 55, Superintendent of Documents, U. S. Government Printing Office, Washington, D. C., 1964; 3rd printing with corrections, 1965. MR 29 \#4914; MR 31 \#1400.

2. J. N. LYNESS, "The calculation of Fourier coefficients by the Möbius inversion of the Poisson summation formula. I," Math. Comp., v. 24, 1970, pp. 101-135.

3. J. N. LYNESS, "Adjusted forms of the Fourier coefficient asymptotic expansion and applications in numerical quadrature," Math. Comp., v. 25, 1971, pp. 87-104.

4. J. N. LYNESS, \& B. W. NinHAM, "Numerical quadrature and asymptotic expansions," Math. Comp., v. 21, 1967, pp. 162-178. MR 37 \#1081.

5. W. E. SMITH \& J. N. LYNESS, "Applications of Hilbert transform theory to numerical quadrature," Math. Comp., v. 23, 1969, pp. 231-252. MR 40 \#5132. 